\title{
Detection of gonioscopically occludable angles and primary angle closure glaucoma by estimation of limbal chamber depth in Asians: modified grading scheme
}

Paul J Foster, Joe G Devereux, Poul Helge Alsbirk, Pak Sang Lee, Davaatseren Uranchimeg, David Machin, Gordon J Johnson, Jamyanjav Baasanhu

\begin{abstract}
Aim-To evaluate the performance of limbal chamber depth estimation as a means of detecting occludable drainage angles and primary angle closure, with or without glaucoma, in an east Asian population, and determine whether an augmented grading scheme would enhance test performance.

Method-A two phase, cross sectional, community based study was conducted on rural and urban areas of Hövsgöl and Ömnögobi provinces, Mongolia. 1800 subjects aged 40 to 93 years were selected and $1717(95 \%)$ of these were examined. Depth of the anterior chamber at the temporal limbus was graded as a percentage fraction of peripheral corneal thickness. An "occludable" angle was one in which the trabecular meshwork was seen in less than $90^{\circ}$ of the angle circumference by gonioscopy. Primary angle closure (PAC) was diagnosed in subjects with an occludable angle and either raised pressure or peripheral anterior synechiae. PAC with glaucoma (PACG) was diagnosed in cases with an occludable angle combined with glaucomatous optic neuropathy and consistent visual morbidity.

Results-Occludable angles were identified in 140 subjects, 28 of these had PACG. The $15 \%$ grade (equivalent to the traditional "grade 1") yielded sensitivity and specificity of $84 \%$ and $86 \%$ respectively for the detection of occludable angles. The 5\% grade gave sensitivity of $91 \%$ and specificity of $93 \%$ for the detection of PACG. The interobserver agreement for this augmented grading scheme was good (weighted kappa 0.76).
\end{abstract}

Conclusions-The traditional limbal chamber depth grading scheme offers good performance for detecting occludable drainage angles in this population. The augmented scheme gives enhanced performance in detection of established PACG. The augmented scheme has potential for good interobserver agreement. (Br F Ophthalmol 2000;84:186-192)

Gorrespondence to:

International Centre for Eye Health, Institute of

Ophthalmology, Bath Street, London EC1V 9EL

Accepted for publication 15 September 1999 for all people suspected of having gar angles and primary angle closure glaucoma in The high prevalence of occludable drainage people of east Asian descent suggests that this should be considered in all adult Asians attending for an ophthalmological assessment. ${ }^{1-4}$ Gonioscopy is considered the definitive method of assessing the characteristics of the drainage angle; however, it is not always well tolerated, and may be time consuming in a busy practice.

Estimation of the limbal chamber depth was suggested as a non-invasive alternative to gonioscopy. A four point grading scheme was shown to give results comparable with a five point gonioscopic grading scheme in estimation of the angular width of the peripheral anterior chamber. ${ }^{5}$ Subsequently, there have been a few descriptions of the performance of this test in populations reported to have a high prevalence of primary angle closure glaucoma. Studies in Greenland, southern India, and Taiwan reported suboptimal performance of the test in screening for primary angle closure. ${ }^{6-8}$ The (presumed) large number of people affected by primary angle closure glaucoma in east and south east Asia ${ }^{9}$ has prompted us to examine the performance of this test in two regions of Mongolia, and to attempt to enhance its performance by modifying the traditional grading scheme.

\section{Subjects and methods}

Ethical approval was given by the ministry of health, Ulaanbaatar, Mongolia. The work was performed in accordance with the tenets of World Medical Association's Declaration of Helsinki. The study was carried out in two regions-Hövsgöl Province, the most northerly region of the country, where 1000 subjects aged 40 years and older were selected for examination in 1995 using a combination of multistage, clustered, simple random, and systematic sampling. ${ }^{3}$ The second phase of the study was carried out in Ömnögobi, the most southerly region of Mongolia, during 1997. Local government census data were used to identify people aged 40 years and older in the towns of Dalanzadgad (the regional capital) and Sevrei. The populations of these towns were stratified by age, after which 560 subjects were selected in Dalanzadgad and a further 240 in Sevrei. The subjects in Ömnögobi were evenly distributed between each decade age group.

The grading of limbal chamber depth (LCD) was carried out at a slit lamp (Model 900 BM, Haag-Streit, Bern, Switzerland). The 


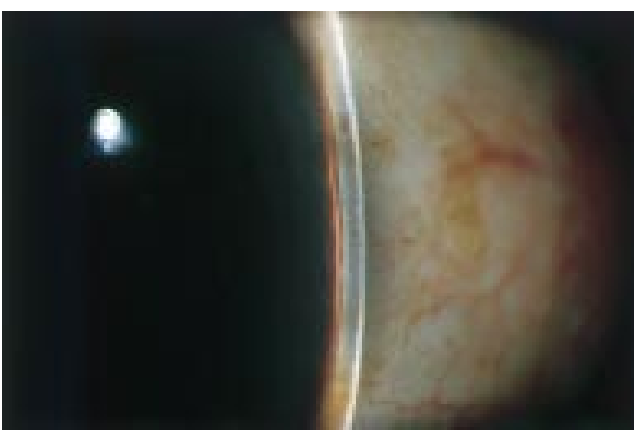

Figure 1 Limbal chamber depth $=5 \%$ of peripheral corneal thickness.

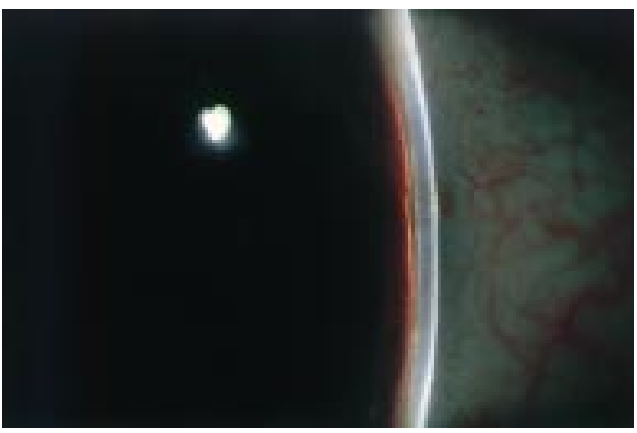

Figure 2 Limbal chamber depth $=15 \%$ of peripheral corneal thickness.

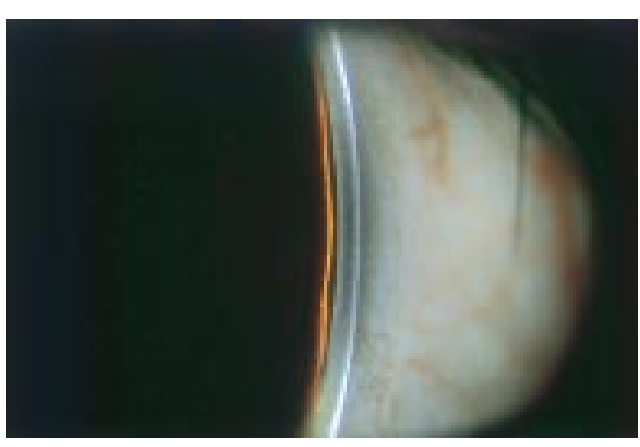

Figure 3 Limbal chamber depth $=25 \%$ of peripheral corneal thickness.

illumination column was offset from the axis of the microscope by $60^{\circ}$. The brightest, narrowest possible vertical beam of light was directed at the temporal limbus, with the beam of light perpendicular to the ocular surface, and viewed from the nasal aspect. The beam was positioned at the most peripheral point of the cornea that would allow a clear view of the anterior chamber and peripheral iris. The objective magnification was set to $\times 1.6$, giving $\times 16$ magnification overall. The LCD was then graded as a percentage fraction of the thickness of the adjacent cornea in the following seven categories: $0 \%, 5 \%, 15 \%, 25 \%, 40 \%, 75 \%$, and $\geqslant 100 \%$. These values were chosen to give class limits of $0 \%,<10 \%,<20 \%,<30 \%,<50 \%$, $<100 \%$, and $\geqslant 100 \%$ respectively, thus allowing comparison with studies using the traditional grades: grade $1<1 / 4$, grade $2=1 / 4$, grade $3=1 / 4-1 / 2$, and grade $4 \geqslant$ full thickness of the peripheral cornea. ${ }^{5}$ Figures 1-6 illustrate the grades $5 \%$ to $\geqslant 100 \%$. The $0 \%$ grade was defined as iridocorneal contact for at least one clock hour in that quadrant (not necessarily continuous).

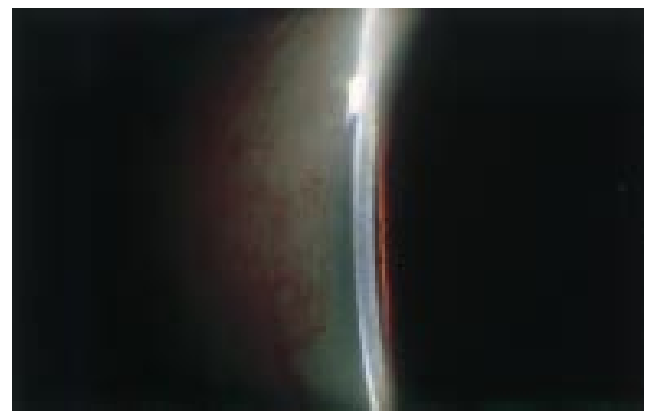

Figure 4 Limbal chamber depth $=40 \%$ of peripheral corneal thickness.

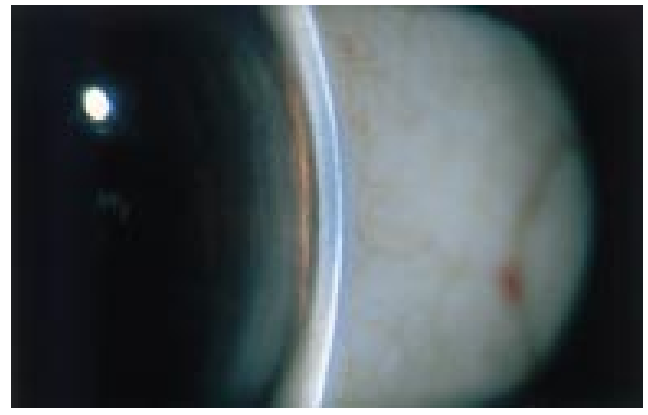

Figure 5 Limbal chamber depth $=75 \%$ of peripheral corneal thickness.

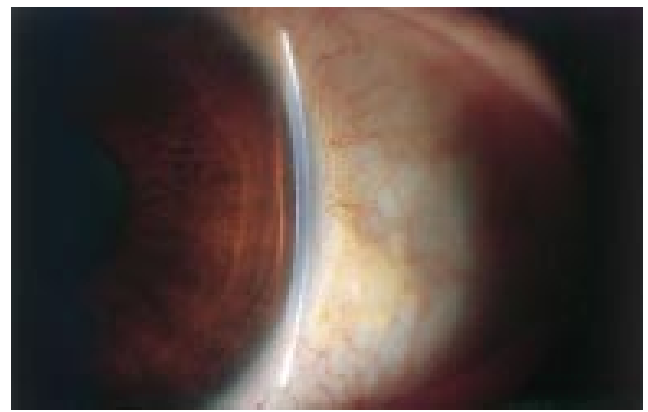

Figure 6 Limbal chamber depth $>100 \%$ of peripheral corneal thickness.

Gonioscopy was attempted during the examination of all subjects using a two mirror gonioscope ("Goldmann" Model, Clement Clarke International, Harlow, Essex). Benoxinate hydrochloride $0.3 \%$ (Moorfields Eye Hospital Pharmacy, London) was used as a corneal anaesthetic. A $2 \%$ hypromellose solution in saline (Moorfields Eye Hospital Pharmacy, London) was used as a coupling medium for the contact lens. Anterior chamber angles were classified as occludable or nonoccludable. An occludable angle was defined as one in which the posterior trabecular meshwork was visible for less than $90^{\circ}$ of the angle circumference, with gaze in the primary position. Slight tilting to gain a view over the convexity of the iris was permitted, but further manipulation of the lens or redirection of gaze was avoided because of the possibility of exerting pressure on the cornea and artificially widening the angle. Primary angle closure (PAC) was diagnosed in those with an occludable drainage angle and another abnormality, but in whom the optic disc and visual field were not felt to be definitely glaucomatous. PAC was diagnosed if at least one of the following 
Table 1 Age and sex specific frequencies of limbal chamber depth

\begin{tabular}{|c|c|c|c|c|c|c|c|c|c|c|}
\hline \multirow[b]{2}{*}{ Sex } & \multirow[b]{2}{*}{ Age } & \multicolumn{8}{|c|}{ Limbal chamber depth* } & \multirow{2}{*}{$\begin{array}{l}\text { Total } \\
\text { subjects }\end{array}$} \\
\hline & & $0 \%$ & $5 \%$ & $15 \%$ & $25 \%$ & $40 \%$ & $75 \%$ & $\geqslant 100 \%$ & Ungraded & \\
\hline \multirow[t]{5}{*}{$\overline{M e n}$} & $40-49$ & 0 & 2 & 19 & 32 & 60 & 61 & 92 & 0 & 266 \\
\hline & $50-59$ & 1 & 11 & 15 & 27 & 52 & 50 & 54 & 4 & 214 \\
\hline & $50-69$ & 4 & 9 & 18 & 30 & 33 & 28 & 21 & 10 & 153 \\
\hline & $70-79$ & 3 & 6 & 8 & 24 & 12 & 10 & 7 & 16 & 86 \\
\hline & $80+$ & 2 & 1 & 0 & 2 & 3 & 1 & 2 & 13 & 24 \\
\hline \multirow[t]{6}{*}{ Women } & $40-49$ & 1 & 14 & 36 & 70 & 107 & 89 & 88 & 1 & 406 \\
\hline & $50-59$ & 8 & 34 & 44 & 69 & 61 & 47 & 17 & 2 & 282 \\
\hline & $60-69$ & 9 & 17 & 30 & 43 & 33 & 18 & 8 & 13 & 171 \\
\hline & $70-79$ & 2 & 13 & 12 & 27 & 14 & 5 & 6 & 12 & 91 \\
\hline & $80+$ & 0 & 3 & 5 & 2 & 7 & 0 & 2 & 5 & 24 \\
\hline & Total & 30 & 110 & 187 & 326 & 382 & 309 & 297 & 76 & 1717 \\
\hline
\end{tabular}

${ }^{\star}$ Limbal chamber depth given as a percentage fraction of peripheral corneal thickness.

Table 2 Comparison of gonioscopic appearance and limbal chamber depth grades

\begin{tabular}{lcccc}
\hline \multirow{2}{*}{$\begin{array}{l}\text { Limbal chamber } \\
\text { depth }\end{array}$} & \multicolumn{2}{l}{ Gonioscopic appearancet } & \\
\cline { 2 - 4 } & Not occludable & Occludable & Ungradable & Total \\
\hline Ungradable & 57 & 11 & 8 & 76 \\
$0 \%$ & 6 & 23 & 1 & 30 \\
$5 \%$ & 52 & 55 & 3 & 110 \\
$15 \%$ & 157 & 30 & 0 & 187 \\
$25 \%$ & 304 & 20 & 2 & 326 \\
$40 \%$ & 379 & 1 & 2 & 309 \\
$75 \%$ & 308 & 0 & 1 & 297 \\
$100 \%$ & 297 & 0 & 0 & 1717 \\
Total & 1560 & 140 & 17 & \\
\hline
\end{tabular}

${ }^{\star}$ Limbal chamber depth was estimated at the temporal limbus as a percentage fraction of the thickness of the adjacent cornea.

†Gonioscopic appearance was classified as occludable if less than $90^{\circ}$ of the total circumference of the posterior trabecular meshwork could be seen without manipulation of the gonioscope.

features was detected: peripheral anterior synechiae, an intraocular pressure $>19 \mathrm{~mm} \mathrm{Hg},{ }^{3}{ }^{10}$ excessive pigment deposition on the superior trabecular meshwork, ischaemic sequelae of acutely raised IOP (distortion of the radially orientated iris musculature, iris stromal atrophy or glaucomfleken), or a clear history of symptoms consistent with sudden IOP rise and evidence of a surgical peripheral iridotomy (PI). In 1995, a darkroom/prone provocation test giving a rise in IOP of $\geqslant 8 \mathrm{~mm} \mathrm{Hg}$ was also used to diagnose PAC.

Primary angle closure glaucoma (PACG) was diagnosed in subjects with an occludable drainage angle, glaucomatous optic neuropathy with consistent visual morbidity. Optic discs were examined through an undilated pupil, unless a satisfactory view could not be obtained, in which case the pupils were dilated. The disc variables of glaucoma were defined as a cup:disc ratio (CDR) of $\geqslant 0.7$ or CDR asymmetry of $\geqslant 0.2$ between the two eyes. All subjects underwent a 26 point static threshold related suprathreshold visual field screening test (Henson CFA 3000, Tinsley Medical Instruments, Newbury, Berks). If abnormal, this was extended to a 66 point test. Subjects with test results suspected of being glaucomatous underwent a confirmatory 56 point threshold visual field test on the same instrument. In mild to moderate cases (CDR $0.7-0.8$, or asymmetry of CDR), a reproducible visual field defect consistent with the pattern of optic nerve damage was required to confirm the diagnosis. In cases with advanced glaucoma (CDR $\geqslant 0.9$ ) a visual field test was attempted, but reliable perimetric quantification of field loss was not an absolute requirement for diagnosis. Eyes in which it was not possible to assess the disc, but which showed ischaemic sequelae of primary angle closure (iris stromal atrophy and sectorial distortion of the radially oriented muscle fibres) and had a visual acuity of $<10 / 200$ were also classified as having PACG. This definition of PACG has been altered slightly from that used in a previous report, ${ }^{3}$ to focus the diagnosis of glaucoma on definite "end organ damage" accompanied by defective visual function.

The clinical grading in these studies was carried out by the one of two investigators (PJF, 1995; JGD, 1997). A comparison of agreement between these two observers for grading of the LCD and angular width of the peripheral anterior chamber was carried out in a consecutive series of 55 eyes of 28 subjects.

The narrowest measurable LCD for the two eyes was taken as the grade for that subject. If it was not possible to allocate a grade for either eye the subject was excluded from the analysis. A subject was classified as having gonioscopically occludable angles if one or both eyes met these criteria. The classification of glaucoma was likewise made regardless of whether one or

Table 3 Performance indices of limbal chamber depth estimation in detection of gonioscopically occludable angles, primary angle closure, and primary angle closure glaucoma

\begin{tabular}{|c|c|c|c|c|c|c|c|}
\hline \multirow[b]{2}{*}{ Limbal chamber depth } & \multirow[b]{2}{*}{ Traditional grade* } & \multicolumn{2}{|c|}{ All occludable angles } & \multicolumn{2}{|c|}{$\begin{array}{l}\text { Primary angle closuret with or } \\
\text { without glaucoma }\end{array}$} & \multicolumn{2}{|c|}{ Primary angle closure glaucoma $\neq$} \\
\hline & & Sensitivity \% & Specificity \% & Sensitivity \% & Specificity $\%$ & Sensitivity \% & Specificity \% \\
\hline $0 \%$ grade & NA & 17.8 & 99.6 & 29.3 & 99.2 & 52.2 & 98.9 \\
\hline$\geqslant 5 \%$ grade & NA & 60.5 & 96.1 & 70.7 & 93.7 & 91.3 & 92.6 \\
\hline$\geqslant 15 \%$ grade & Grade 1 & 83.7 & 85.7 & 84.5 & 82.4 & 91.3 & 81.1 \\
\hline$\geqslant 25 \%$ grade & Grade 2 & 99.2 & 65.5 & 100 & 62.4 & 100 & 61.1 \\
\hline$\geqslant 40 \%$ grade & Grade 3 & 100 & 40.3 & 100 & 38.3 & 100 & 37.5 \\
\hline
\end{tabular}

*Traditional "van Herick" grades. ${ }^{5}$

†Primary angle closure, those subjects with angles judged to be occludable on gonioscopic examination with either elevated intraocular pressure, peripheral anterior synechiae or a positive dark room, prone provocation test.

$\ddagger$ Primary angle closure glaucoma- those subjects with an occludable drainage angle and glaucomatous optic neuropathy, corroborated by visual field evidence in mild or moderate cases. 
Table 4 Positive and negative predictive values of the test for limbal chamber depth estimation in detection of gonioscopically occludable angles, primary angle closure, and primary angle closure glaucoma

\begin{tabular}{|c|c|c|c|c|c|c|c|}
\hline \multirow[b]{2}{*}{ Limbal chamber depth } & \multirow[b]{2}{*}{ Traditional grade } & \multicolumn{2}{|c|}{ All occludable angles* } & \multicolumn{2}{|c|}{$\begin{array}{l}\text { Primary angle closuret with or } \\
\text { without glaucoma }\end{array}$} & \multicolumn{2}{|c|}{ Primary angle closure glaucoma } \\
\hline & & $P P V T \%$ & NPVT \% & PPVT \% & NPVT \% & PPVT \% & NPVT \% \\
\hline $0 \%$ grade & NA & 76.4 & 94.3 & 57.0 & 97.5 & 27.7 & 99.6 \\
\hline$\geqslant 5 \%$ grade & NA & 53.1 & 97.1 & 28.9 & 98.9 & 9.0 & 99.9 \\
\hline$\geqslant 15 \%$ grade & Grade 1 & 29.9 & 98.6 & 14.8 & 99.3 & 3.7 & 99.9 \\
\hline$\geqslant 25 \%$ grade & Grade 2 & 17.3 & 99.9 & 8.8 & 100 & 2.0 & 100 \\
\hline$\geqslant 40 \%$ grade & Grade 3 & 10.8 & 100 & 5.5 & 100 & 1.3 & 100 \\
\hline
\end{tabular}

^Prevalence $6.8 \%$

†Primary angle closure, those subjects with angles judged to be occludable on gonioscopic examination with either elevated intraocular pressure, peripheral anterior synechiae or a positive dark room, prone provocation test with or without glaucoma. Prevalence $3.5 \%$.

$\ddagger$ Primary angle closure glaucoma, those subjects with an occludable drainage angle and glaucomatous optic neuropathy, corroborated by visual field evidence in mild or moderate cases. Prevalence $0.8 \%$.

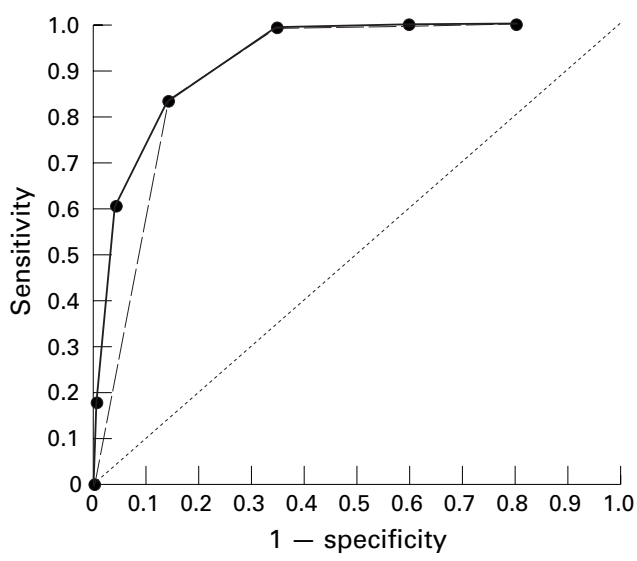

Figure 7 Receiver operating curve comparing traditional and augmented limbal chamber depth grading in the detection of individuals with a drainage angle judged to be occludable on gonioscopic examination. Solid line indicates performance of augmented grading scheme. Broken line indicates the performance of the traditional grading scheme. Dotted line indicates the plot for a test with a predictive value equal to that of chance.

both eyes were affected. The units of analysis were therefore subjects, not eyes. The efficacy of LCD grading as a screening tool was assessed by calculating sensitivity, specificity, positive and negative predictive values of the test. ${ }^{11}$ In calculating the latter two variables, the following prevalence figures were used: occludable angles, $6.8 \%$; PAC, $3.5 \%$; PACG, $0.8 \%$ in the population aged 40 years and older. ${ }^{3}$ Receiver operating characteristic (ROC) curves were used to compare traditional and augmented grading schemes. The area under the curve is an indicator of test performance. The ROC analysis was carried out using a commercially available software package (True Epistat, Epistat Services, Richardson, TX, USA). The interobserver agreement was assessed by calculating the weighted kappa statistic. $^{12}$

Table 5 Area under the receiver operating characteristic (ROC) curves for the traditional and augmented tests

\begin{tabular}{llc}
\hline & \multicolumn{2}{l}{ Area under ROC curve $(95 \%$ confidence intervals) } \\
\cline { 2 - 3 } & Traditional test & Augmented test \\
\hline Occludable drainage angles & $0.898(0.879,0.916)$ & $0.928(0.910,0.946)$ \\
Primary angle closure & $0.883(0.858,0.908)$ & $0.925(0.899,0.951)$ \\
Primary angle closure glaucoma & $0.888(0.854,0.922)$ & $0.955(0.922,0.988)$ \\
\hline
\end{tabular}

*Traditional "van Herick" grades. ${ }^{5}$

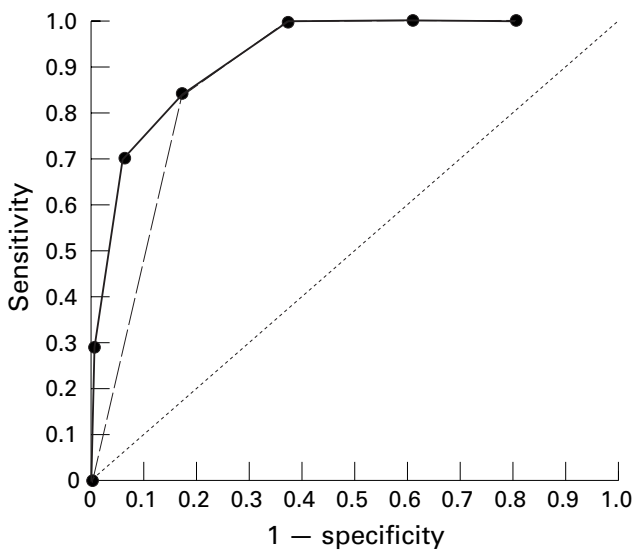

Figure 8 Receiver operating curve comparing traditional ${ }^{5}$ and augmented limbal chamber depth grading schemes in the detection of primary angle closure. Primary angle closure was diagnosed in those subjects with angles judged to be occludable on gonioscopic examination and either elevated intraocular pressure, peripheral anterior synechiae, or a positive dark room, prone provocation test. This group includes those people with glaucomatous optic neuropathy. Solid line indicates performance of augmented grading scheme. Broken line indicates the performance of the traditional grading scheme. Dotted line indicates the plot for a test with a predictive value equal to that of chance.

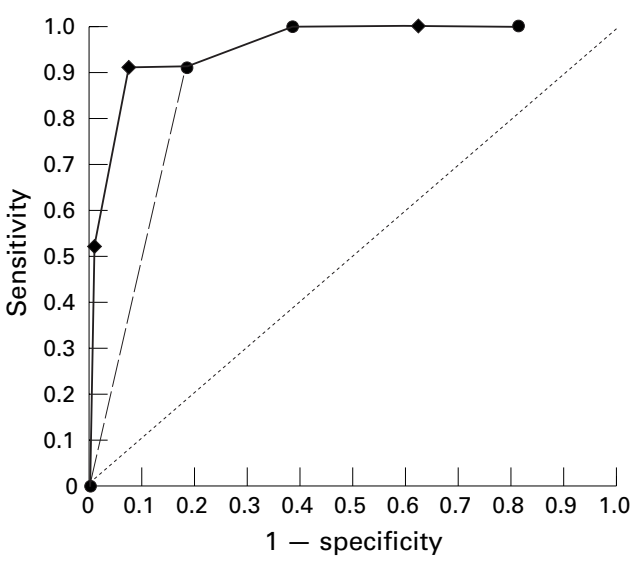

Figure 9 Receiver operating curve comparing traditional 5 and augmented limbal chamber depth grading schemes in the detection of primary angle closure glaucoma. Primary angle closure glaucoma was diagnosed in those subjects with an occludable drainage angle and glaucomatous optic neuropathy, corroborated by visual field evidence in mild or moderate cases. Solid line indicates performance of augmented grading scheme. Broken line indicates the performance of the traditional grading scheme. Dotted line indicates the plot for a test with a predictive value equal to that of chance. 
Table 6 The performance of limbal chamber depth estimation in detection of primary angle closure

\begin{tabular}{|c|c|c|c|c|c|c|c|}
\hline Location & N America ${ }^{17}$ & Greenland $^{6}$ & Australia $^{18}$ & India $^{7}$ & Taiwan $^{8}$ & Mongolia & \\
\hline Setting & Clinic & Community & Clinic & Clinic & Community & Community & \\
\hline Subjects & 135 & 311 & 1113 & 96 & 562 & 1717 & \\
\hline$L C D / P C T^{\star}$ cut off & $\leqslant 1 / 4$ & $\leqslant 1 / 4$ & $\leqslant 0.2$ & $<1 / 4$ & $1 / 4$ to $1 / 2$ & $\leqslant 25 \%$ & $\leqslant 5 \%$ \\
\hline Sensitivity & $24 / 29(83 \%)$ & $51 / 56(91 \%)$ & $24 / 27(89 \%)$ & $13 / 21(62 \%)$ & $15 / 16(94 \%)$ & $139 / 140(99 \%)$ & $26 / 28(91 \%)$ \\
\hline Specificity & $89 / 106(84 \%)$ & $136 / 255(53 \%)$ & $3 / 1086(99 \%)$ & $67 / 75(89 \%)$ & $282 / 487(58 \%) \quad 461 / 487(95 \%)$ & $1022 / 1560(66 \%)$ & $1564 / 1689(93 \%)$ \\
\hline "Gold standard" & $\begin{array}{l}\mathrm{ACD} \leqslant 2.00 \\
\mathrm{~mm}\end{array}$ & $\begin{array}{l}\mathrm{ACD} \leqslant 2.00 \\
\mathrm{~mm} \dagger\end{array}$ & Gonioscopy $\star \star$ & Gonioscopy & Diagnosis of "PACG" $\ddagger$ & Gonioscopyt† & $\begin{array}{l}\text { Gonioscopy, disc } \\
\text { and field } \\
\text { examination㧊 }\end{array}$ \\
\hline
\end{tabular}

${ }^{\star} \mathrm{LCD} / \mathrm{PCT}=$ the ratio of depth of the anterior chamber at the temporal limbus to the adjacent peripheral corneal thickness used as the criterion of screening test failure.

$\star \star$ Gonioscopy was carried out only if LCD/PCT $\leqslant 0.3$.

†Known cases of primary angle closure excluded.

†Primary angle closure glaucoma: diagnosed irrespective of disc damage or visual field loss, if angle appeared narrow on gonioscopic examination and the intraocular pressure $>18 \mathrm{~mm} \mathrm{Hg}$ or positive dark room prone provocation test. Three subjects with previous attacks of acute angle closure treated by iridectomy were included. ††For detection of occludable drainage angle as defined in text. Gonioscopy was not possible in 17 people.

$\ddagger \neq$ For detection of occludable drainage angle and glaucomatous visual morbidity.

\section{Results}

From the total of 1800 subjects selected in the two regions, 1717 (95.4\%) were examined. A gonioscopically occludable drainage angle was found in at least one eye of 140 subjects. Thirty five people were classified as having PAC, and a further 28 met the criteria for PACG. Table 1 summarises the age and sex of the subjects, together with the distribution of LCD grades. Table 2 compares the number of subjects with and without occludable angles, and their LCD grade. Table 3 gives details of sensitivity and specificity of LCD estimation in predicting the presence of an occludable drainage angle, PAC (with or without glaucoma), and PACG only. Table 4 summarises the predictive values of the test. Figures 7-9 are receiver operating characteristic (ROC) curves comparing the performance of the traditional and augmented tests in the detection of all occludable angles, PAC (with or without glaucoma), and PACG respectively. Table 5 gives the area under each of these curves (together with 95\% confidence intervals). The area under the curve was greater for the augmented test in all instances, indicating superior test performance, although the confidence intervals did overlap in the curves for PAC and occludable drainage angles, indicating that these may have occurred by chance. In practical terms, the major difference between the two grading schemes appears to be in the detection of primary angle closure glaucoma (subjects with occludable drainage angles and evidence of glaucomatous visual morbidity). The point at which the augmented test plot approaches the top left hand corner of the graph (the point that indicates a perfect test performance) most closely in Figure 9 represents the $5 \%$ test grade, with sensitivity and specificity of $91 \%$ and $93 \%$ respectively. The agreement between the two observers was good (weighted kappa $=0.76$ ) for LCD grading, and very good (weighted kappa = 0.80 ) for assessment of angular width of the drainage angle.

\section{Discussion}

The original description of limbal chamber depth (LCD) estimation as a method of predicting the width of the drainage angle was based on data from 5436 patients, aged from the first to the seventh decade, attending a practice in San Francisco for refraction.
Approximately 400 of this number were examined by gonioscopy. It was stated that the four point LCD grading scheme was roughly equivalent to another five point method of grading the gonioscopic estimate of the angular width of the peripheral anterior chamber. ${ }^{13}$ There was rarely disagreement by more than one grade, and if disagreement did occur, the gonioscopic estimate was usually narrower than the LCD grade. ${ }^{5}$

The population distribution of LCD has subsequently been studied in Europe, North America, and Asia. LCD was graded as a percentage fraction of peripheral corneal thickness (PCT) in the Framingham Eye Study. In this predominantly white North American population, $6 \%$ of people had LCD $\leqslant 30 \% .{ }^{14}$ In Rotterdam, Netherlands, only $2.2 \%$ of a group of 6760 subjects aged 55 years and older were found to have LCD $\leqslant 25 \%$ PCT. $^{15}$ Surprisingly, a study in Tibet found a similar figure; $2.4 \%$ of people aged 40 years and older had LCD $\leqslant 1 / 4$ PCT. The prevalence of primary angle closure glaucoma in this east Asian population was reported to be very low. ${ }^{16}$ The efficacy of the technique as a screening tool was not evaluated in these studies. By comparison, $40 \%$ of Mongolians had LCD $\leqslant 1 / 4$ PCT (Table $1 ; 653 / 1641$ ). It does appear that European derived populations have substantially fewer people with shallow anterior chambers at the limbus, despite the higher proportion of elderly people.

Table 6 compares data from Mongolia with the results of previous studies examining the efficacy of LCD estimation as a method of detecting primary angle closure. The current study of 1717 adults in a community based setting provides new data for the east Asia region, where PACG is believed to be a major cause of visual morbidity. ${ }^{138}$ Our observations were validated by gonioscopic examination in all subjects, unlike the original description of this technique. Previous reports detailed in Table 6 suggest that LCD estimation does not combine high sensitivity and specificity in other populations. Our findings indicate that the test does have the potential to perform well in the detection of gonioscopically occludable angles. Using the traditional "grade 1" (LCD $\leqslant 15 \%$ PCT), $84 \%$ of such subjects would be detected. If "grade 2" (LCD $\leqslant 25 \%$ PCT) is used the sensitivity rises to $99 \%$. 
The choice of grade used to determine screening test failure depends on the purpose of the examination. We envisage this test as having two potential applications. Many ophthalmologists in Asia do not have access to a gonioscope or sufficient training in the technique. The LCD technique may present an opportunity for these doctors to carry out opportunistic screening in their clinic. Patients with narrow angles could be identified before they had developed glaucoma, and referred for definitive examination in a unit with more sophisticated equipment. An ideal test would have a high sensitivity. This would generate a certain number of false positive test results, but should virtually exclude false negatives (when a potentially pathological state may be classified as normal). Use of the traditional "grade 2" (LCD $\leqslant 25 \%$ PCT) would miss one subject in 100 with an occludable angle, if this test were used in isolation. Grade 3 (LCD $\leqslant 40 \%$ ) would identify all cases. We do not suggest that the use of estimation of LCD be used instead of gonioscopic examination of patients suspected of having glaucoma.

The second potential role for this technique is in population screening for PACG, possibly using a hand held slit lamp. In such a programme, the LCD grade used to indicate a "screening failure" would be determined by economic and logistic imperatives, as well as statistical considerations. The natural history of primary angle closure in an asymptomatic phase is poorly understood. Hence the choice of strategy (to detect eyes with occludable angles without "end organ damage", or those with established disease) and the optimal timing and nature of intervention are still to be determined. However, if the aim were to detect eyes with established optic nerve damage and visual field loss, LCD estimation appears to perform well. Using the LCD $\leqslant 5 \%$ PCT grade would give $91 \%$ sensitivity and $93 \%$ specificity. When considering the suitability of a test for mass screening, the prevalence of the condition should also be considered. The positive and negative predictive values of the test (PPVT and NPVT) take this factor into account. Thus, in our study population (aged 40 years and over), a person with a positive test at the LCD $\leqslant 5 \%$ PCT grade would have a $1 / 11$ probability of having PACG. A negative test would incorrectly classify $1 / 1000$ people as being normal. The aim of screening is usually to detect a condition in its early stages, before significant damage (meaning visual loss, in the case of glaucoma) has occurred. We have therefore used the term "glaucoma" to indicate those people with established visual loss, and "primary angle closure" to indicate a disorder of anterior segment physiology or anatomy without visual morbidity. When screening for PACG, the aim would be detection of PAC or an occludable drainage angle, in order to give prophylactic treatment (presumably a laser iridotomy). In the detection of occludable drainage angles, the $5 \%$ and $15 \%$ LCD grades gave a PPVT of $53 \%$ and $30 \%$ and a NPVT of $97 \%$ and $99 \%$ respectively. Using the traditional grade 1 (LCD $\leqslant 15 \%$ ) as the criterion for fail- ure of the screening examination, one in three people with positive test result would be correctly identified as having an occludable drainage angle, and one person in 100 would be incorrectly classified as normal.

One unforeseen factor that limited the efficacy of this test in the Mongolian population was the unusually high prevalence of climatic droplet keratopathy (Foster, unpublished data, 1995). The deposition of a plasma protein-like material in and under Bowman's membrane causes reduced clarity of the cornea, typically most marked at the limbus. Another unquantified factor that doubtless degrades the performance of the test is variation of the position of the limbus. Reported asymmetry of the LCD grade between temporal and nasal aspects may be a reflection of this. ${ }^{6}$ All our measurements were made at the temporal limbus at the most peripheral location where a clear view was possible.

Our findings disagree with those from southern India, where the test performed poorly in a clinic based setting. The only explanation we can offer for this finding is that it may be a reflection of a differing mechanism underlying angle closure in the two populations. It may be that the Indian population are less prone to pupil block (felt to be the predominant mechanism in Mongolia), and consequently have a less anterior bowing of the mid-peripheral iris. However, we did share the finding of this study that the technique shows good interobserver reproducibility. ${ }^{7}$

In summary, we have found that the traditional method of assessing and grading limbal chamber depth offers a sensitive method of detecting occludable drainage angles in east Asians. The use of a modified grading scheme offers enhanced performance if the aim were to detect established cases of primary angle closure glaucoma.

This study was funded by the British Council for Prevention of Blindness with additional support from Danida (Copenhagen), the International Glaucoma Association (London), and The Danish Association for the Blind (Copenhagen). Analysis and writing was facilitated by grants from the National Medical Research Council, Singapore and the Singapore National Eye Centre.

Proprietary interests: None.

$1 \mathrm{Hu} Z$, Zhao ZL, Dong FT. An epidemiological investigation of glaucoma in Beijing and Shun-yi county. [Chinese] Chung-Hua Yen Ko Tsa Chih [Chinese fournal of Ophthatmology] 1989;25:115-18.

2 Salmon JF, Mermoud A, Ivey A, et al. The prevalence of primary angle closure glaucoma and open angle glaucoma in Mamre, Western Cape, South Africa. Arch Ophthalmol Mamre, Western

3 Foster PJ, Baasanhu J, Alsbirk PH, et al. Glaucoma in Mongolia-a population-based survey in Hövsgöl Province, Northern Mongolia. Arch Ophthalmol 1996;114: 1235-41

4 Nguyen N, Mora JS, Gaffney MM, et al. A high prevalence of occludable angles in a Vietnamese population. Ophthalmology 1996;103:1426-31.

5 Van Herick W, Shaffer RN, Schwartz A. Estimation of the width of the angle of anterior chambers: incidence and significance of the narrow angle. Am F Ophthalmol 1969;68: 626-9.

6 Alsbirk PH. Limbal and axial chamber depth variations. A population study in eskimos. Acta Ophthalmol 1986;64: 593-600.

7 Thomas R, George T, Braganza A, et al. The flashlight test and van Herick's test are poor predictors for occludable angles. Aust NZ $\mathcal{F}$ Ophthalmol 1996;24:251-6.

8 Congdon N, Quigley HA, Hung PT, et al. Screening techniques for angle-closure glaucoma in rural Taiwan. techniques for angle-closure gla
Acta Ophthalmol 1996;74:113-19. 
9 Quigley HA. Number of people with glaucoma worldwide. Br f Ophthalmol 1996;80:389-93.

10 Foster PJ, Baasanhu J, Alsbirk PH, et al. Central corneal thickness and intraocular pressure in a Mongolian population. Ophthalmology 1998;105:969-73.

11 Altman DG, Bland JM. Diagnostic tests. 2: Predictive values. BMF 1994;309:102.

12 Altman DG. Practical statistics for medical research. London: Chapman and Hall, 1991.

13 Becker B, Shaffer RN. Diagnosis and therapy of the glaucomas. St Louis: CV Mosby, 1965.

14 Leibowitz HM, Krueger DE, Maunder LR. The Framingham Eye Study Monograph. Surv Ophthalmol 1980;Suppl 24:335-610.

15 Wolfs RCW, Grobbee DE, Hofman A, et al. Risk of acute angle-closure glaucoma after diagnostic mydriasis in non-selected subjects: the Rotterdam Study. Invest Ophthalmol Vis Sci 1997;38:2683-7.

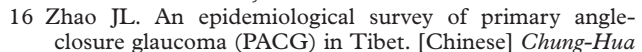
Yen Ko Tsa Chih [Chinese fournal of Ophthalmology] 1990;26:47-50.

17 Vargas E, Drance SM. Anterior chamber depth in angle-closure glaucoma. Clinical methods of depth determination in people with and without the disease. Arch Ophthalmol 1973;90:438-9.

18 Cockburn DM. Slit lamp estimate of anterior chamber ysiol Opt 1982:59:904-8. 